\title{
Formation of Samin Diastereomers by Acid-catalyzed Transformation of Sesamolin with
}

\section{Hydrogen Peroxide}

Authors: Hsin-Ya Tsai ${ }^{1, \S}$, Wei-Ju Lee ${ }^{2,3, \S}$, I-Hsuan Chu ${ }^{1}$, Wei-Ching Hung ${ }^{1}$, and Nan-Wei Su${ }^{1 *}$

${ }^{1}$ Department of Agricultural Chemistry, National Taiwan University, Taipei 10617, Taiwan

${ }^{2}$ School of Food Safety, Taipei Medical University, Taipei 11042, Taiwan

${ }^{3}$ Master program in Food Safety, Taipei Medical University, Taipei 11042, Taiwan

*Corresponding author:

*Nan-Wei Su, Ph.D., Professor

Department of Agricultural Chemistry, National Taiwan University, Taipei 10617, Taiwan

Tel: +886-2-33664806

Fax: +886-2-23632714

E-mail: snw@ntu.edu.tw 

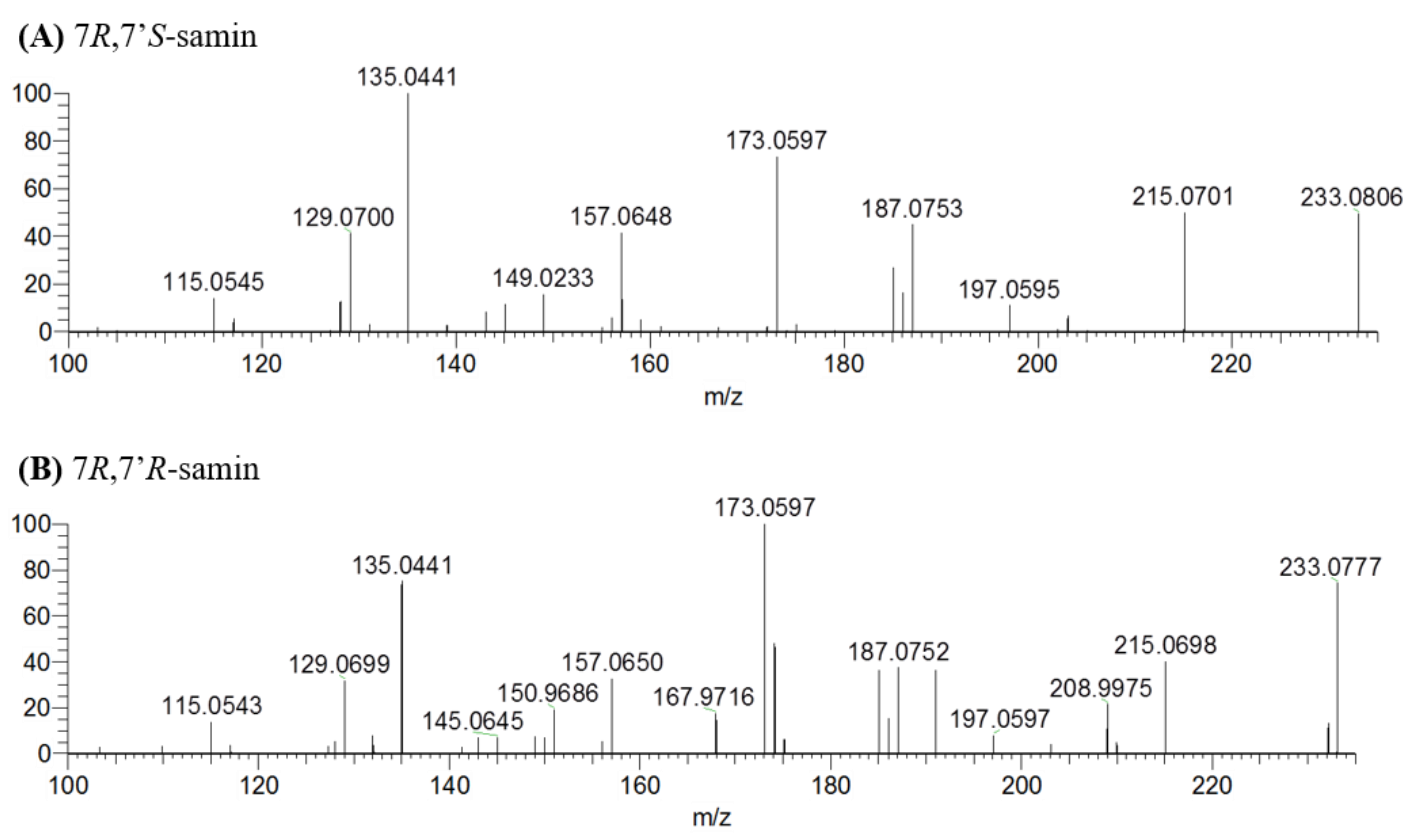

Supplementary Figure 1. ESI-MS spectra of protonated $7 R, 7^{\prime} S$-samin (a) and $7 R, 7^{\prime} S$-samin (b) fragment ions. 
UV-Vis spectrum of compound b (7R,7'R-samin) :

$\lambda \max =204,235,287 \mathrm{~nm}$

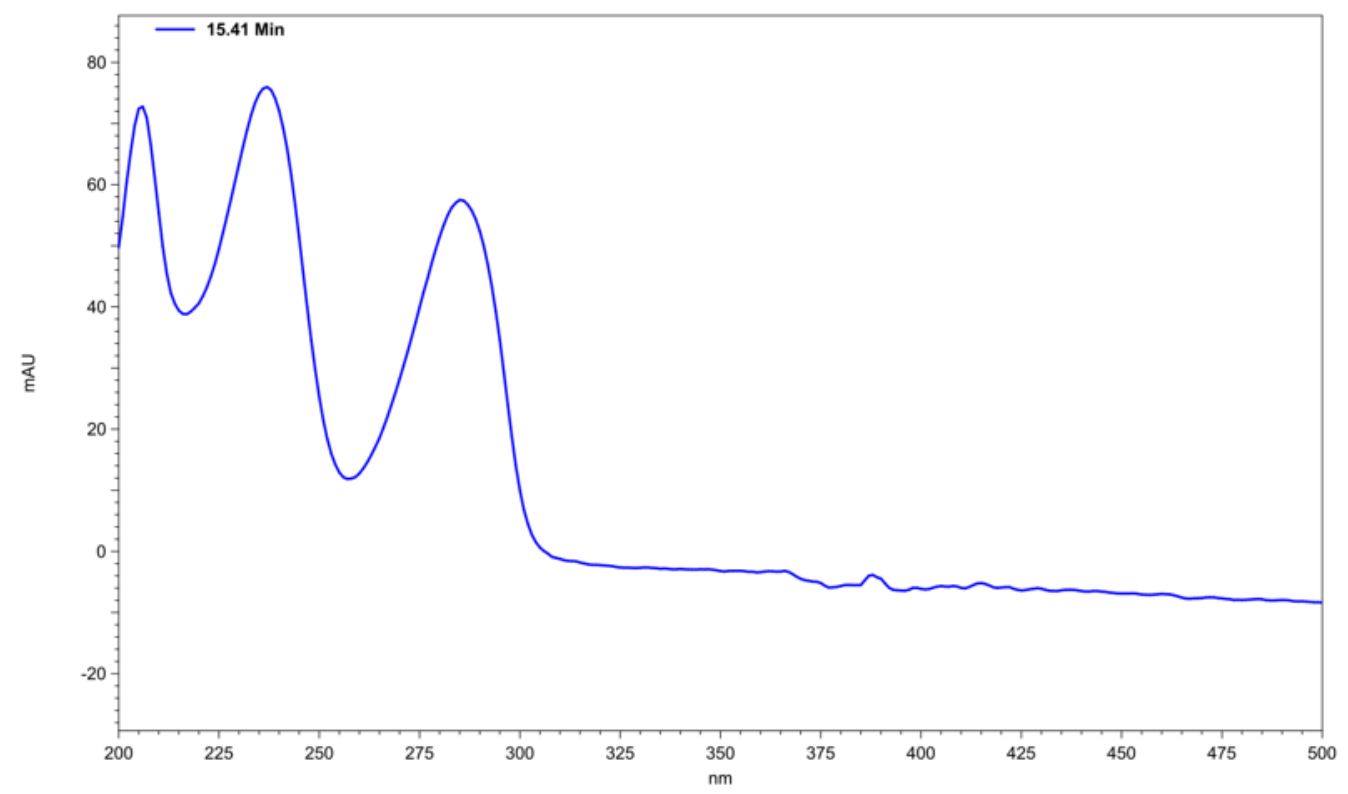

Supplementary Figure 2. UV-Vis spectrum of compound b (7R,7'R-samin) at $200-500 \mathrm{~nm}$. 


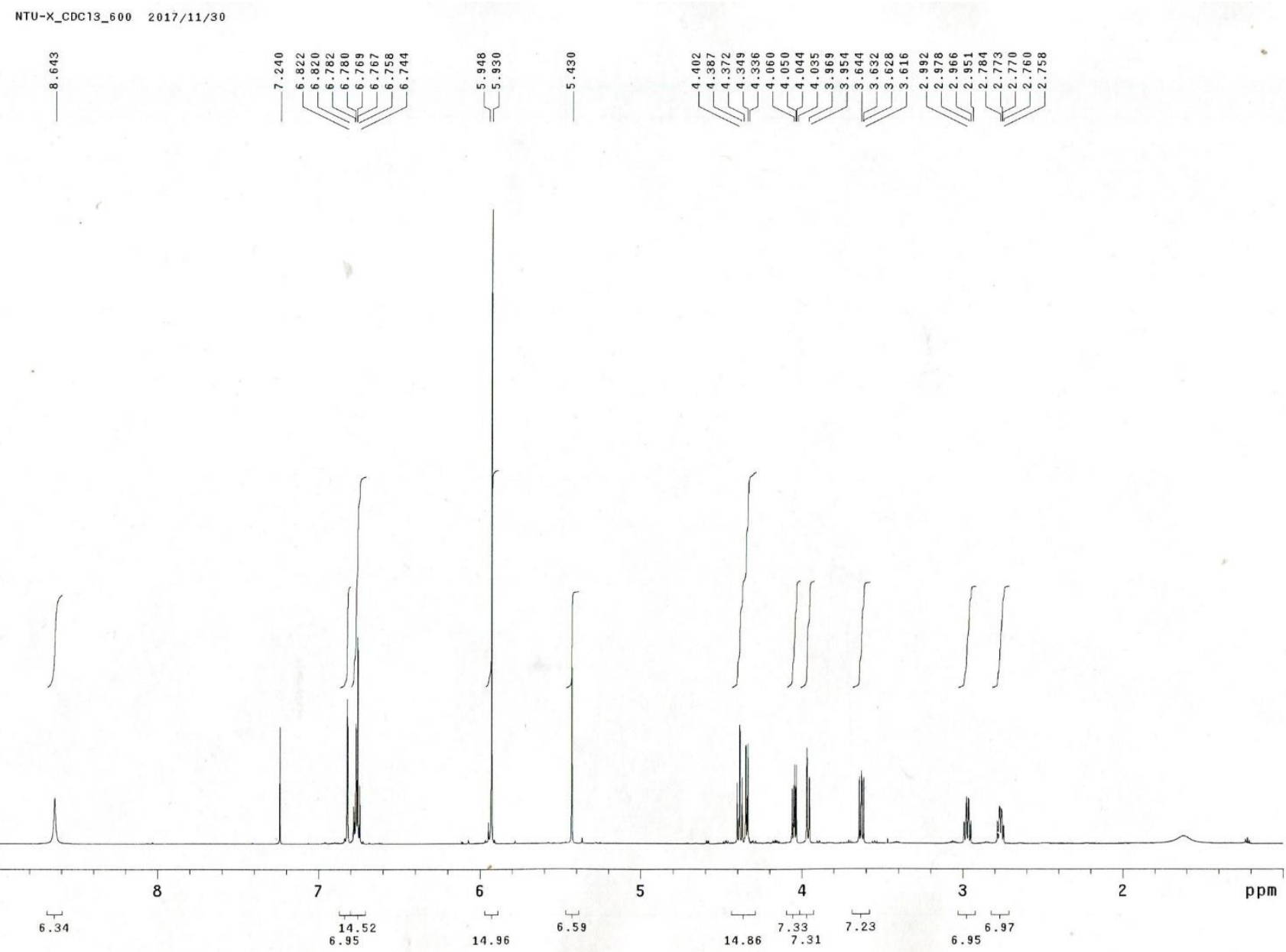

Supplementary Figure 3. ${ }^{1} \mathrm{H}$ spectrum of compound $\mathbf{b}\left(7 R, 7^{\prime} R\right.$-samin $)$. 
NTU-X_CDC13_600 2017/11/30

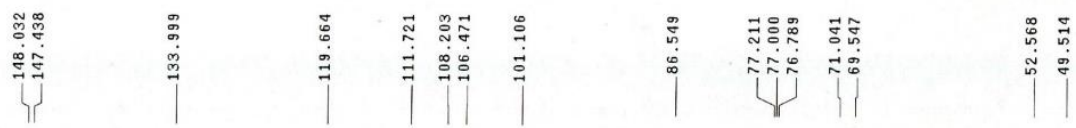

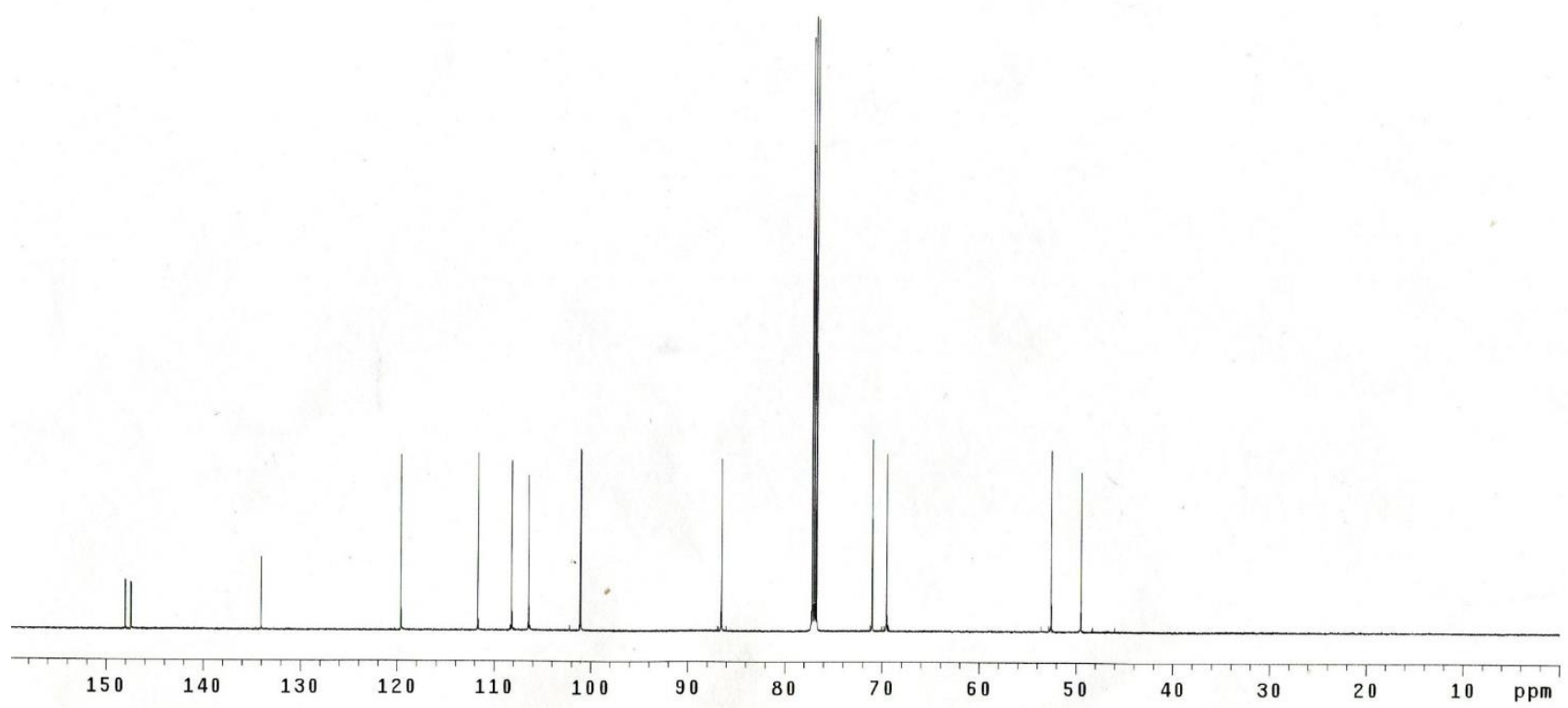

Supplementary Figure $4 .{ }^{13} \mathrm{C}$ spectrum of compound $\mathbf{b}\left(7 R, 7^{\prime} R\right.$-samin). 


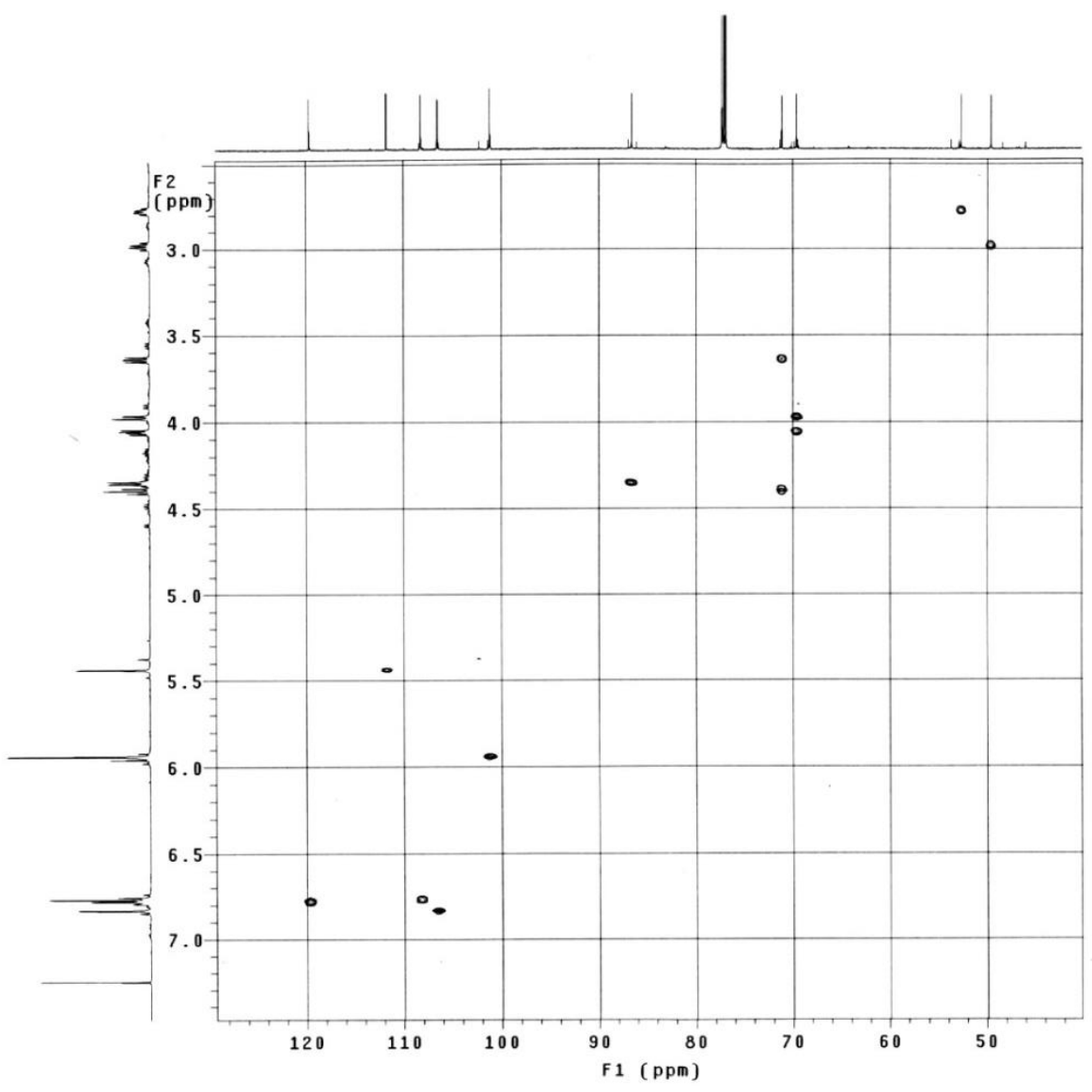

Supplementary Figure 5. HSQC spectrum of compound $\mathbf{b}\left(7 R, 7^{\prime} R\right.$-samin). 


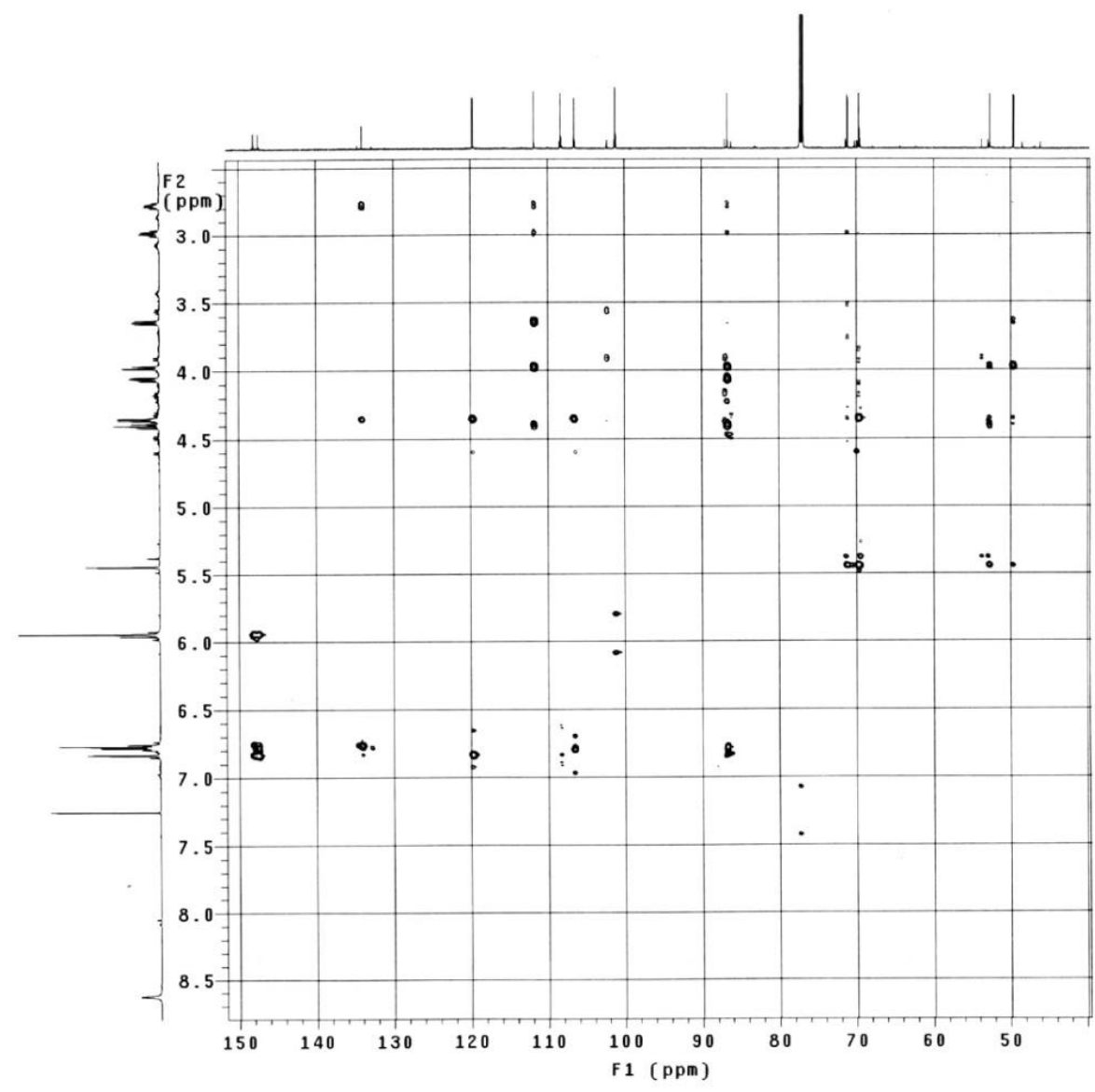

Supplementary Figure 6. HMBC spectrum of compound $\mathbf{b}\left(7 R, 7^{\prime} R\right.$-samin). 


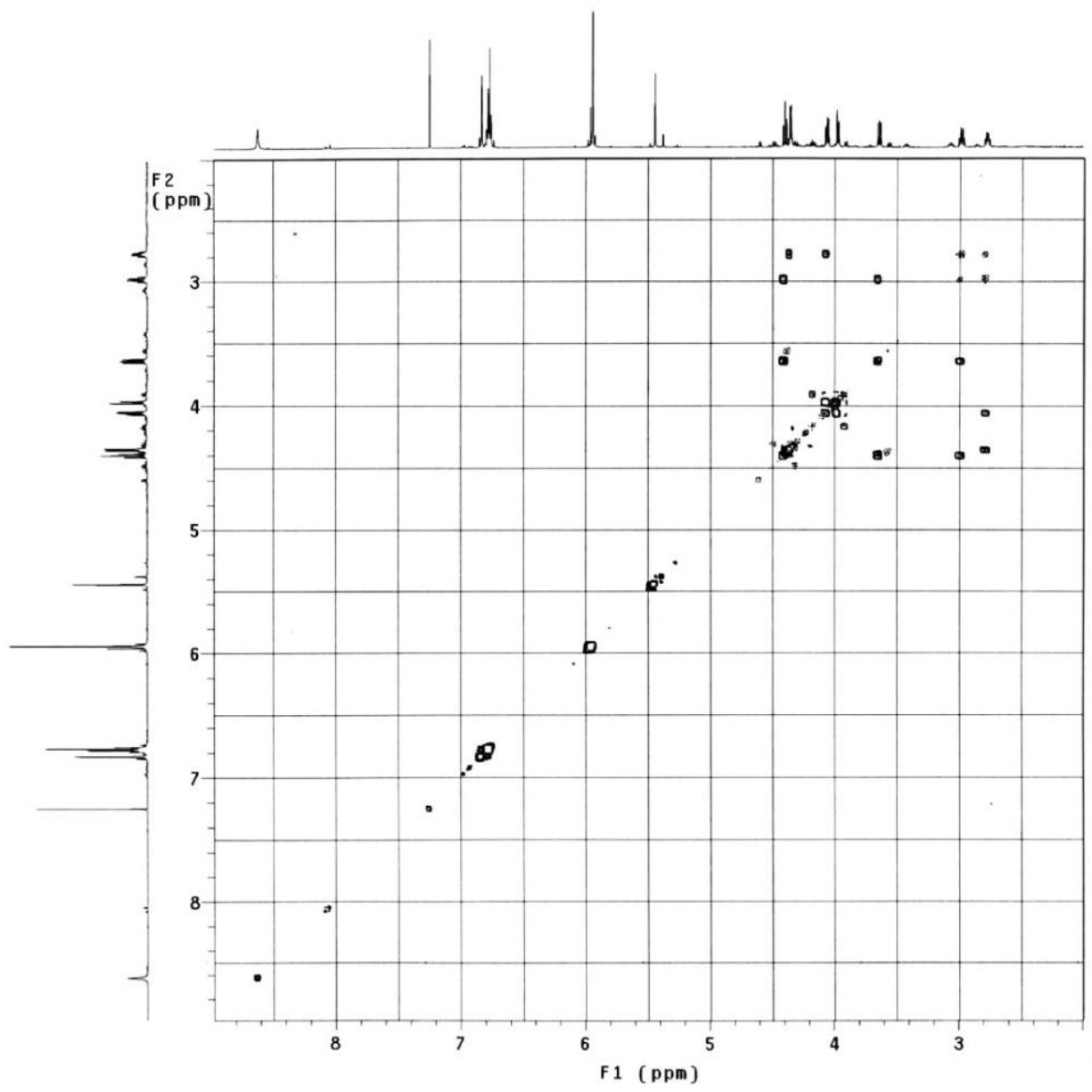

Supplementary Figure 7. COSY spectrum of compound $\mathbf{b}\left(7 R, 7^{\prime} R\right.$-samin). 


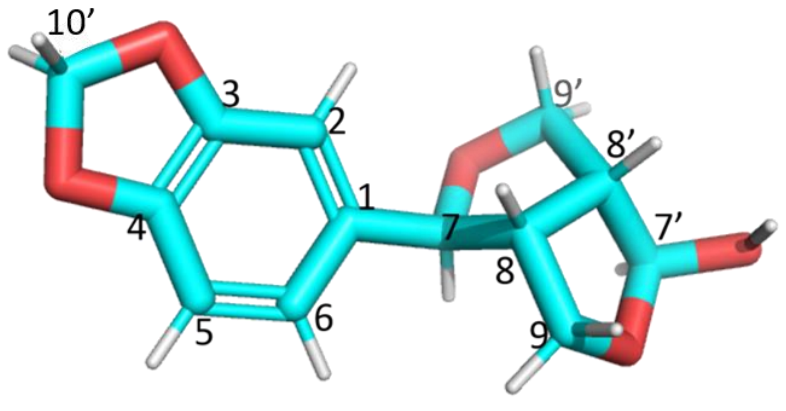

$7 R, 7^{\prime} S$-samin

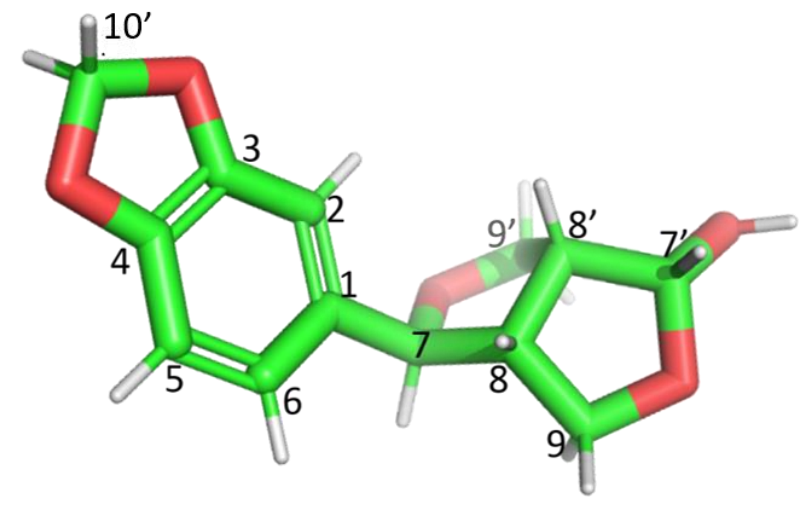

$7 R, 7^{\prime} R$-samin

Supplementary Figure 8. Stereo structures of compound a ( $7 R, 7^{\prime} S$-samin) and compound b

$\left(7 R, 7^{\prime} R\right.$-samin). 